\title{
Implementing Sustainable International Accepted Trainings at Muhima Hospital, Kigali, Rwanda
}

\author{
Saideth Gato ${ }^{1}$, Rondi Anderson ${ }^{1}$ \\ ${ }^{1}$ Muhima Hospital, Kigali, Rwanda
}

\section{Background}

This is a description of a pilot project that was implemented at the Muhima Hospital, the largest maternity hospital in Rwanda. The goal of the program was to facilitate the teaching of staff the best practice of maternal child health. The program was established through monthly staff trainings using three evidenced-based global maternal and neonatal health programs: Helping Babies Breath, Helping Mothers Survive, and Basic Life Support.

\section{Methods}

The "Train the Trainer Model" was used and monthly staff trainings were developed and implemented. The students were experienced nursing staff trained by advanced midwifery students during their clinical rotation at Muhima Hospital. Human Resources for Health (HRH) educational mentors supported the students and staff during the trainings. Following the first training, Muhima staff nurses were selected based on expertise and enthusiasm to be future trainers. These nurses received advanced instruction as trainers. The long-term program goal is that all hospital staff and students complete each of these three training programs once every two years.

\section{Results}

Initially the staff expressed resistance to the process, but this barrier was overcome as the staff became comfortable and effective as providers and trainers. Trainings done in the local language elicited more participation compared to trainings in English. Staff and students enjoyed the simulations and developed a comfort level and camaraderie between staff, students and HRH educators.

\section{Conclusions}

Challenges of sustainability in relation to motivation and organizational issues is being discussed with the hospital director and the staff trainers. Planning and organizing are ongoing, with the goal to expand the program to include health centers. This professional staff development project and the desire for community outreach programs are impressive. Community outreach would be beneficial for the professional development of the nurse midwife trainers and staff in the rural district facilities throughout Rwanda.

Key words: helping babies breath, helping mothers survive, basic life support, teaching, training, Rwanda 